\title{
Candida albicans Pyomyositis in a Patient with Systemic Lupus Erythematosus
}

YU-HISU CHEN, MD; CHUN-CHI LU, MD; HSIANG-CHENG CHEN, MD, PhD, FACR, Division of Rheumatology/Immunology/Allergy, Department of Internal Medicine, Tri-Service General Hospital, National Defense Medical Center, Taipei, Taiwan, R.O.C. Address correspondence to Dr. H.C. Chen, Chief, Division of Rheumatology/Immunology/Allergy, Department of Internal Medicine, Tri-Service General Hospital, National Defense Medical Center, No. 325 , Sec. 2, Cheng-Gong Rd., Neihu 114, Taipei, Taiwan, R.O.C.E-mail: hccheng@ndmctsgh.edu.tw. Ethics board approval was not required in accordance with the policy of the authors' institution. Patient consent was obtained. J Rheumatol 2018;45:286-7; doi:10.3899/jrheum.170122

Fungal pyomyositis is a rare intramuscular infection characterized by swelling and pain at the site of infection. The most common fungal pathogen is Candida spp ${ }^{1}$. The risk factors include immunodeficiency, previous fungal infection, and broad-spectrum antibiotic exposure ${ }^{2}$. In addition, steroid therapy may induce subclinical myopathy and subsequent infectious myositis ${ }^{3}$.

A 48-year-old man presented with intermittent pain and swelling of the lower limbs for 1 month. He was diagnosed with Candida albicans fungemia and received oxacillin and fluconazole for 2 weeks. He had systemic lupus erythematosus (SLE) with secondary Sjögren syndrome complicated by pulmonary hypertension, Child-Pugh class B liver cirrhosis related to hepatitis B infection and alcoholism, and type 2 diabetes mellitus. Previous treatments included hydroxychloroquine, prednisolone $10 \mathrm{mg} /$ day, and methylprednisolone $80 \mathrm{mg} /$ day for 3 days bimonthly because of SLE flares. Physical examination revealed hyperpigmentation, local heat, grade 3 edema of the lower limbs, and bilateral palpable pulsation of the dorsalis pedis. Computed tomography of the lower limbs showed lobulated cystic lesions of varying size in the bilateral flexor hallucis and flexor digitorum muscles (Figure 1). He underwent fasciotomy, and histological examination showed necrotizing inflammation with pseudohyphae and spores within muscle (Figure 2). Tissue culture yielded $C$. albicans. He underwent debridement and 6-week micafungin treatment, which resulted in complete remission.

Chronic lower limb swelling may be seen in patients with SLE; however, if it coexists with pain and other risk factors of pyomyositis, diagnostic imaging could be helpful for the identification of underlying infection.

\section{REFERENCES}

1. Wong CH, Chang HC, Pasupathy S, Khin LW, Tan JL, Low CO. Necrotizing fasciitis: clinical presentation, microbiology, and determinants of mortality. J Bone Joint Surg Am 2003;85:1454-60.

2. Crum-Cianflone NF. Bacterial, fungal, parasitic, and viral myositis. Clin Microbiol Rev 2008;21:473-94.

3. Tsai SH, Peng YJ, Wang NC. Pyomyositis with hepatic and perinephric abscesses caused by Candida albicans in a diabetic nephropathy patient. Am J Med Sci 2006;331:292-4.

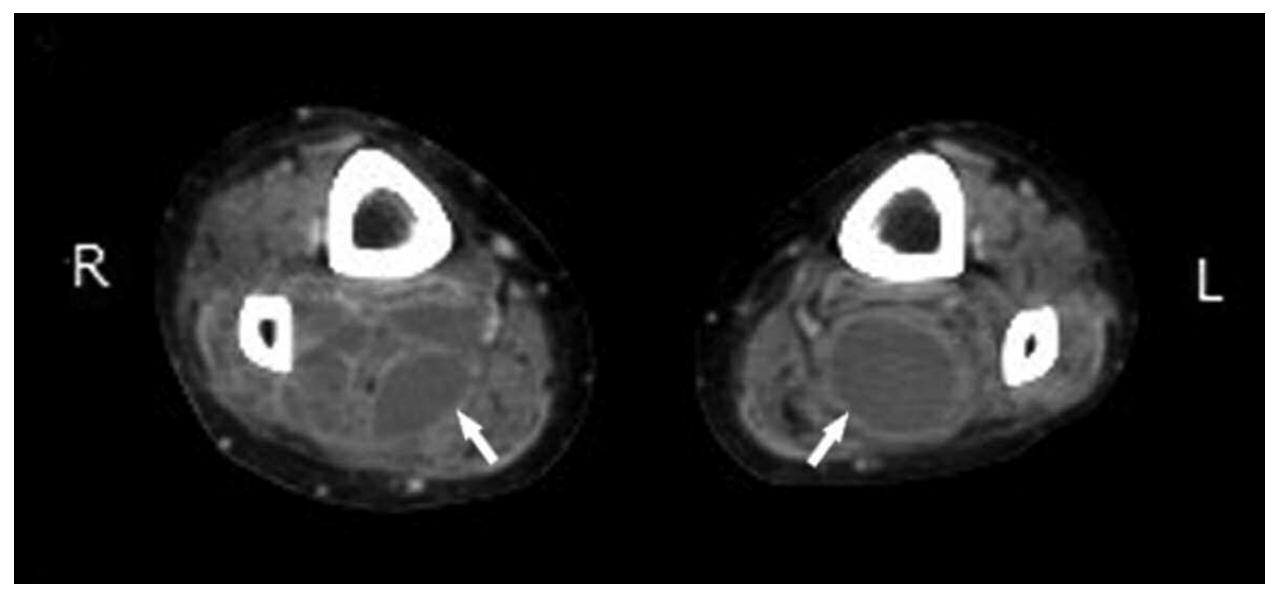

Figure 1. Computed tomography shows lobulated cystic lesions (arrows) of varying size in the bilateral flexor hallucis and flexor digitorum. 


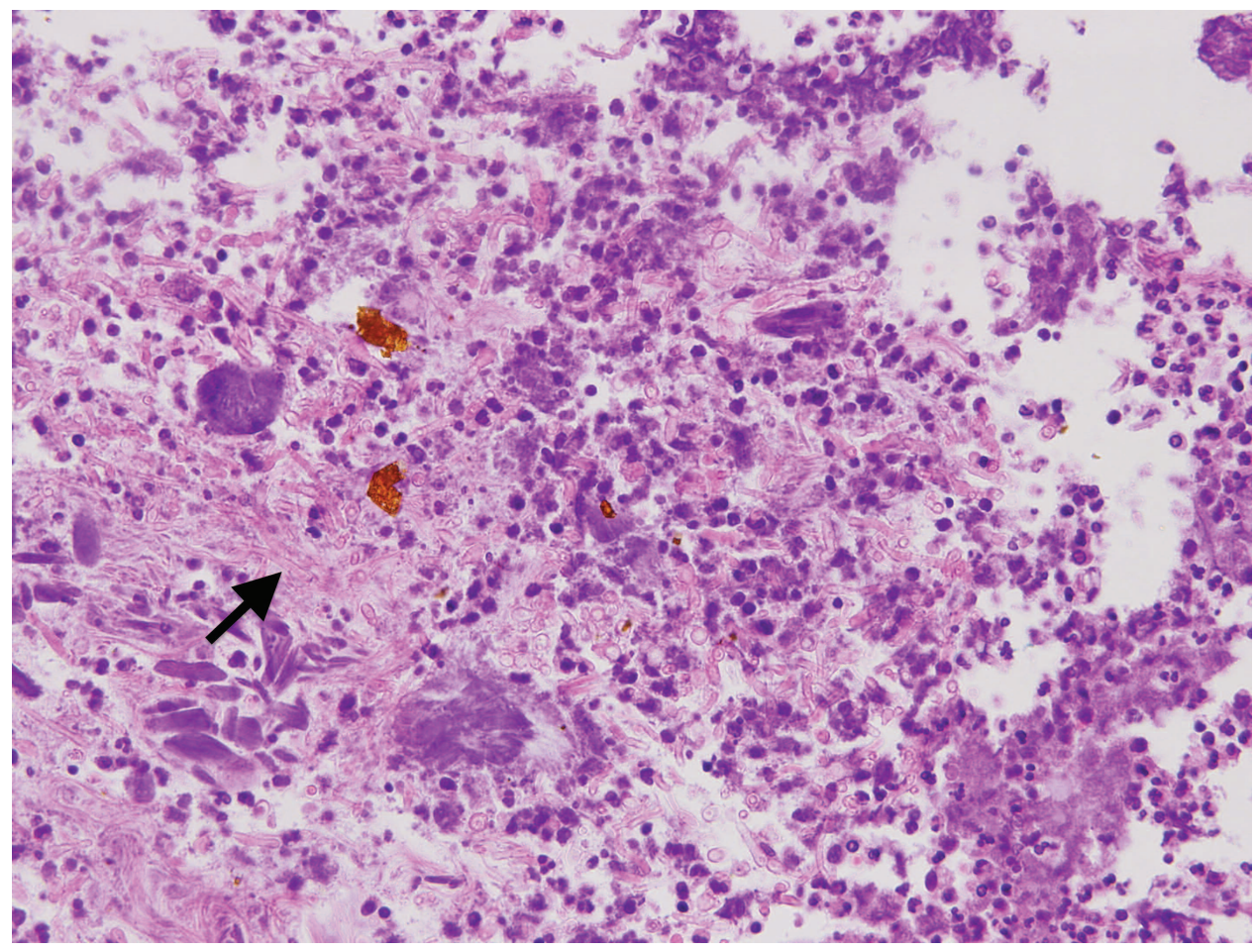

Figure 2. Photomicrograph shows H\&E staining of necrotizing inflammation with pseudohyphae (arrow) and spores within muscle. Magnification $\times 400$. 